\title{
CN Tower Lightning Current Derivative Heidler Model Analysis and Transmission
}

\author{
Ouarda Nedjah $^{1,2}$, Ali M. Hussein ${ }^{1}$, Sridhar Krishnan ${ }^{1}$, Reza Sotudeh ${ }^{2}$ \\ ${ }^{I}$ Electrical and Computer Engineering Department, Ryerson University, Toronto, Ontario \\ ${ }^{2}$ Dept. of Electronic, Comp. \& Elec. Eng., Hertfordshire University, Hertford, UK
}

\begin{abstract}
The lightning current data collected at the CN Tower can be used for calibrating the Lightning Detection Network (LDN) systems. They can also be used in the modification of existing models and the creation of new ones used for the conversion of the lightning current to electromagnetic and electric propagated fields. The measured lightning current derivative signals at the $C N$ tower are noise-laden. They are first denoised. Then, related Heidler models that can be representative for the measured lightning currents discharged through the Tower are generated. The calculated models parameters are provided to the LDN systems to be used in the calibration of the systems. Due to the complexity of the lightning discharge process, Heidler models can't be representative for all the lightning currents waveform. This paper will discuss Heidler model representation of the lightning current measured at the CN Tower and the related problems.
\end{abstract}

\section{Introduction}

Lightning is defined as a transient high current electric discharge. It occurs when some region of the atmosphere gains such a large charge that the electric fields associated with it can cause an electric breakdown of the air [1]. These transient high currents reaching the earth can be devastating to modern society's infrastructures. They frequently cause blackouts and they can destroy or interrupt the operations of communication networks, aircrafts, spacecrafts and electric and electronic devices. The necessity of protection from lightning hazards has made the lightning phenomenon an important area of research since the seventies.

As a result to the phenomenon of erecting high-rise buildings booming in the seventies, people started to experience difficulties receiving their broadcasted TV programs. In order to remedy this problem, the Canadian National $(\mathrm{CN})$ Tower (one of the world's tallest manmade free-standing structure with a height of $553 \mathrm{~m}$ ) was built in 1976 as a telecommunication hub. Since then, the CN Tower has been used as a broadcasting transmission facility, transmitting through its antennas, in both the UHF and VHF bands, more than 20 channels in Toronto.

Lightning strikes to the Tower have been observed since 1978. Since the beginning of the summer of 1991, several measurement stations have been operational to simultaneously capture the lightning current derivative at the $\mathrm{CN}$ Tower, the lightninggenerated electric and magnetic fields, the flash trajectory images viewed from two orthogonal directions, and the return-stroke velocity [2-4].

When analyzing a $\mathrm{CN}$ Tower lightning current waveform, we are interested in the determination of its waveform parameters namely, the wavefront peak, the maximum wavefront steepness, the $10-90 \%$ risetime to the peak and the pulse width at the 50\% level of the peak. Statistical information concerning these waveform parameters is essential to the development of lightning protection measures of power systems, telecommunication networks and sensitive electronic devices.

The extraction of lightning current waveform parameters was made difficult and less accurate by the existence of different kinds of noise in the recorded lightning current derivative data. The noise includes a DC offset (which may be due to the measurement system), high frequency components, low frequency components oscillating in the vicinity of $100 \mathrm{kHz}$ and the interference of reflected currents due to structural discontinuities of the Tower. After a brief description of the lightning current derivative measurement system, a typical $\mathrm{CN}$ Tower current derivative signal will be presented. The current waveform parameters to be extracted are described and the effect of noise on these parameters is analyzed. 
The measured lightning current derivative waveform and its associated current obtained by the integration of the measured signal are denoised by Divide-and-Conquer technique (DAC). The parameters of the current waveform are calculated and provided to an optimization unit that estimates Heidler function parameters that are used to generate an optimum Heidler model associated to the measured lightning current derivative waveform [5]. Unfortunately, these generated models can't be representative for all measured current derivative waveforms. This problem will be discussed in the intention of providing the LDN stations [6] with only heidler models that satisfy certain criteria.

\section{Current derivative measurement system}

A lightning current derivative measurement system was installed at the CN Tower in 1990. It consists of a 3-m (two 1.5 m-long sections) Rogowski sensing coil having a $40-\mathrm{MHz}$ bandwidth with a sensitivity of $0.35 \mathrm{~V} /(\mathrm{A} / \mathrm{ns})$. The coil is placed at the $474-\mathrm{m}$ above ground level (AGL) and is connected via a 146-m triaxial cable to a recording system located at the 372 m AGL.

The current derivative recording system consists of a computer controlled $10 \mathrm{ns,} \mathrm{10-bit,} \mathrm{two-channel}$ digitizer (Tektronix 710 A). It has a capacity of 128 kilobytes of memory per channel, which enables the recording of up to 8 return strokes in a lightning flash, each lasting $164 \mu \mathrm{s}$.

\section{Current derivative signal}

A lightning flash may contain many strokes. Every stroke is a transient high current electric discharge pulse. The Rogowski coil measures a voltage signal that is proportional to the lightning current derivative.

This signal, resulting from a lightning return stroke, is registered in 16 kilobytes of memory by the recording system at a sampling frequency of $100 \mathrm{MHz}$. It forms what is called the return-stroke current derivative waveform. Fig. 1 presents a typical lightning return-stroke current derivative signal, measured at the CN Tower on July 03, 1998 at 17:31 pm. The corresponding lightning current is obtained by numerical integration (Fig. 2).

As seen in Figs. 1-2, the current derivative and its integral signals are corrupted by different kinds of noise, partly due to the initial function of the $\mathrm{CN}$ Tower as a transmission facility (high-frequency noise).
Some of the noise is thought to be due to the measurement system itself, like the DC component. Current reflections due to the tower's structural discontinuities add other interfering noise. Furthermore, low frequency components oscillating in the vicinity of $100 \mathrm{kHz}$ are always visible in the recorded signals. These low frequency noise components have been proved to be a result of Loran-C signals [7]. As a result of the noise, different frequencies are found in the current derivative waveform.

Depending on the current peak and the current wavefront steepness, the return-stroke lightning current pulse may be distinguishable, slightly exceeding the noise level, or completely embedded in noise [8,9]. Due to the presence of different noise components, Figs. 1-2 demonstrate the difficulty of extracting the current waveform parameters, namely, the wavefront peak, the maximum wavefront steepness, the 10-90\% risetime to the peak and the pulse width at the $50 \%$ level of the peak. In some cases, when the current wavefront steepness and/or the current peak are low, most current waveform parameters, if not all, are impossible to determine.

Several methods have been applied to denoise the lightning current derivative signals captured at the $\mathrm{CN}$ Tower from the associated noise. We can mention the Linear Filtering, the Fourier Transform based spectral subtraction, the Adaptive Wavelet Transforms and the optimization based strategies methods DAC that will be described later [8-12].

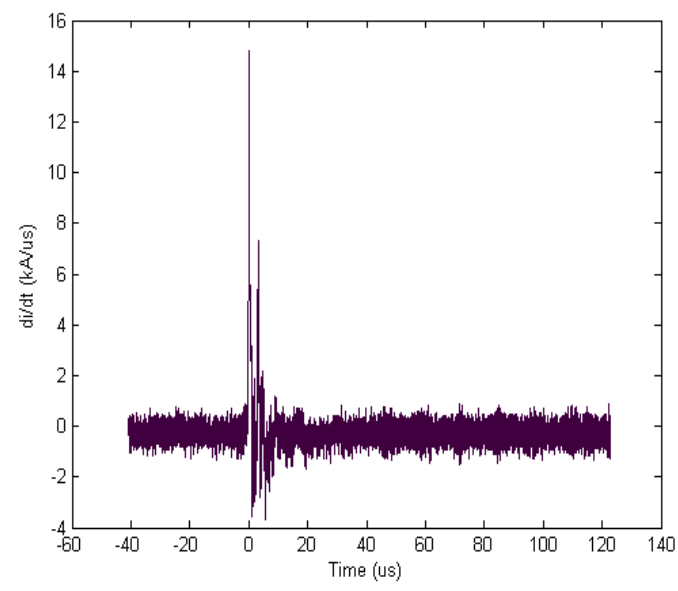

Figure 1. Typical measured current derivative waveform 


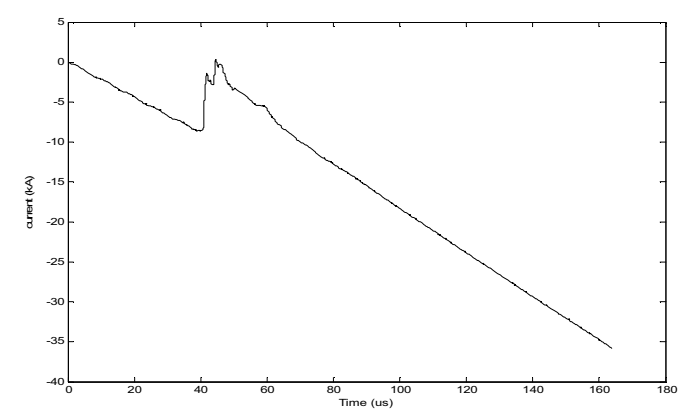

Figure 2. Current waveform

\section{Heidler model and its parameters Calculation}

\subsection{Heidler lightning current modelisation}

Heidler has developed an empirical form for representing a typical lightning current waveform. This formula has been used in many lightning current related models, including the modified transmission (MTL) model treating lightning strikes to tall structures [5].

The Heidler function is given by the following expression:

$$
i(t)=\frac{I_{1}}{2 c_{1}}\left[\frac{\left(t / \tau_{1,1}\right)^{k_{1}} e^{-t / \tau_{2,1}}}{1+\left(t / \tau_{1,1}\right)^{k_{1}}}\right]+\frac{I_{2}}{2 c_{2}}\left[\frac{\left(t / \tau_{1,2}\right)^{k_{2}} e^{-t / \tau_{2,2}}}{1+\left(t / \tau_{1,2}\right)^{k_{2}}}\right]
$$

Where $I_{i}$ is the current peak, $\left(t / \tau_{1, i}\right)^{k} /\left(1+\left(t / \tau_{1, i}\right)^{k}\right.$ represents the current rise function and $e^{\left(-t / \tau_{2}, i\right)}$ corresponds to the current decay function. $\tau_{1, \mathrm{i}}$ and $\tau_{2, \mathrm{i}}$ are the time constants determining the current rise and decay times, respectively, $\mathrm{k}_{\mathrm{i}}>1$ is a current steepness factor and $c_{i}<1$ is a correction factor [13].

After denoising the measured current derivative waveform, some of the calculated parameters of the current waveform are injected together with Heidler function in a curve fitting process to generate an appropriate model. The generated model represents the measured lightning current derivative with an infinite SNR.

\subsection{Lightning current derivative denoising by $\mathrm{DAC}$ and lightning current parameters calculation}

As already stated, the measured lightening current derivative signals are contaminated by a wide range of frequencies including a DC component. The removal of the DC part is the first step in the pretreatment of the waveform. Then, the range of high frequencies is reduced by an adaptive wavelet transform. This process reduces the number of zero crossings in the current derivative waveform. Since the current waveform is obtained by integration, the high frequency noise is automatically removed from this waveform, which makes the search for the local minima and local maxima on the waveform much easier.

After these preliminary processes, the Divide-andConquer process starts based on the initial and final conditions of the lightning signal and the NewtonRaphson optimization technique [14].

The lightning current derivative is divided into three sections; a) the time before the lightning signal, b) the time during the lightning signal, and c) the time after the lightning signal.

As initial and final conditions, it is known that there is no lightning signal before the first appearance of the return stroke; as 20 ( 40 or 80 ) $\mu$ s of the total $160 \mu$ s of the signal duration are registered before the triggering process takes place, the portion of signal just before this time is equalized to zero.

After the return stroke, other than the peaks reflected from the discontinuities of the tower there should be no more peaks in the lightning current or its derivative, and the current should keep on decaying until reaching the zero value after some milliseconds, so after approximately 10 to $20 \mu \mathrm{s}$ the current derivative signal can be replaced by its mean value, hence reducing more noise.

The maxima and the minima on the current derivative waveform correspond to the maximum steepness of the current waveform respectively on ascending and descending curvatures, and the maxima and the minima on this latter one correspond to the zero-crossings on the current derivative waveform as stated by Newton-Raphson technique. This is why the zero-crossings on the current derivative waveform together with the successive minima and maxima on the current waveform on the active period of the waveforms are tracked simultaneously by marching on the two graphs or set of data. The maximum current derivative peak is localized and the first zero crossing of the waveform after it is searched. Its position corresponds to the first current peak position which matches the lightning channel discharge through the tower or what is called the lightning current wavefront peak.

The maximum peak of the current waveform corresponds to the reflection from the ground. Other small peaks in between the wavefront peak and the ground reflection peak are due to the reflections from the Observation Deck levels of the tower. Without the 
reflections from the tower discontinuities, the current would have been decaying until reaching zero. This is why the peaks following the first one that are due to the reflections at the discontinuities have to be removed. There are two major reflection points: the reflection from the top of the Observation Deck and the reflection from the Ground. The reflection parameter can be written as the ratio $\mathrm{R}=\mathrm{E}_{\mathrm{r}} / \mathrm{E}_{\mathrm{i}}$, where $\mathrm{E}_{\mathrm{i}}$ represents the incident waveform amplitude and $E_{r}$ is the reflected waveform amplitude. Once the first and the second successive highest peaks are localized, the reflection parameters are estimated and the portion of signals related to the reflected waveforms are removed by subtraction. After the current waveform is cleaned, its parameters are calculated [11].

Together with the Heidler formula (1), the first three calculated parameters ( the current wavefront peak, the $10-90 \%$ rise time to the peak, its $90-10 \%$ decay time) are injected as initial guesses for the Heidler function parameters in a curve fitting program that is based on the least squares (LSQR) method. The result of optimization represent the Heidler model associated to the lightning current striking the $\mathrm{CN}$ Tower.

Hence, for every measured lightning current derivative waveform, we can obtain a model that can be considered as a clean lightning current waveform with an infinite SNR and that can be represented only by its 10 optimized Heidler function parameters.

Only the 10 Heidler parameters can be transmitted to the LDN stations instead of a lengthy noisy measured waveform. The Heidler model associated to these parameters can be generated on the LDN stations for its use in their system calibration or magnetic and electric field's propagation modeling.

Not all the calculated models match well the measured lightning current derivative waveforms from which they have been generated as it will be shown in the results presented in the next Section. Because of this, it is primordial to ensure that the model peak, the model rise time and its width at $50 \%$ from the peak match the ones of the measured waveform to ensure that the model that will be generated to calibrate the system corresponds exactly to the striking lightning current.

\section{Results}

The signals, represented by Figures 1 and 2 have been denoised by the DAC technique and the result of denoising is represented on Figures 3 and 4. Once the noise associated to the measured current derivative and its integral waveforms was reduced it was possible to calculate their parameters. Tables I and II present these parameters for the current derivative and the current of the signal of Figures 1 and 2.

The denoised current waveform, the minimal, and maximal permissible values of the lightning current model parameters provided by [13] in Table 1, the calculated lightning current parameters (the wavefront peak value, its rising and falling times) provided as initial guesses for Heidler parameters, together with Heidler function formula, are injected into an optimization program. The program consists of a curve fitting process based on the LSQR method. It estimates the Heidler model parameters of the measured waveform based on its initial calculated parameters.

The estimated current Heidler model (that can be considered as the perfect lightning current free of noise) of the signal of Figure 2 and its associated current derivative model obtained either by evaluating the derivative of Heidler model using the estimated heidler model parameters or simply by differentiating the optimized Heidler current model are shown in Figure 3 and 4. The estimated Heidler function parameters representing the measured current derivative waveform measured at the tower that can be transmitted to the LDN stations to be used for their system calibration or propagation models estimation are represented in Table 3.

Unfortunately not all the generated models can match exactly the measured waveforms as shown by Figures 5, 6, 7 and 8. While in Figures 5 and 6 the current waveform matches its model, the current derivative model does not match its measured counterpart as the model represents an average of two successive simultaneous strikes (one arriving before the end of the first one). For Figures 7 and 8 neither the current, nor the current derivative matches exactly their corresponding generated models (maximum peaks), as the current waveform is drowned in the noise. This mismatch between the measured waveforms and their generated models are due to the complexity of the lightning phenomenon: sometimes, before the lightning channel is fully discharged, another charge is added to the channel through other branches of the stepped leader or another stroke from another flash joins the initial stroke as for the case of the signal of Figures 5 and 6 or, in other cases, the current waveform is drowned in the noise and it become difficult to find the correct model, as it is the case for the signal of Figures 7 and 8 . The problem of mismatch of the estimated models with the measured waveforms puts extra constraints on the data to be sent to the LDN stations for calibrating their systems. These constraints impose for the model's parameters to match the ones for the 
measured waveform such as the wavefront peak, the rise time to the peak and the width at $50 \%$ of the peak.

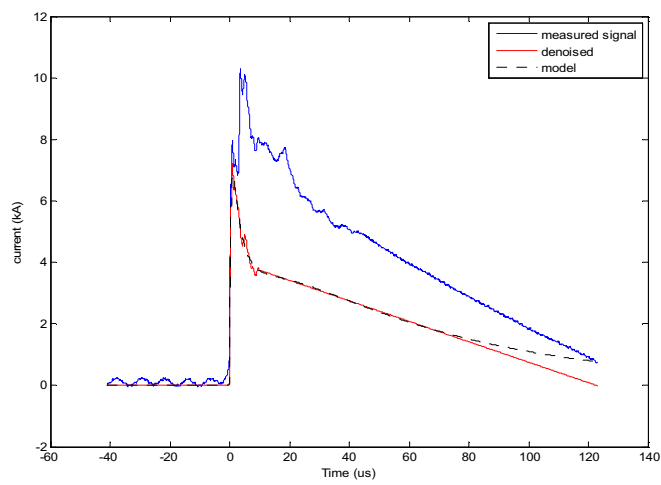

Figure 3. Denoised current waveform and its Heidler model

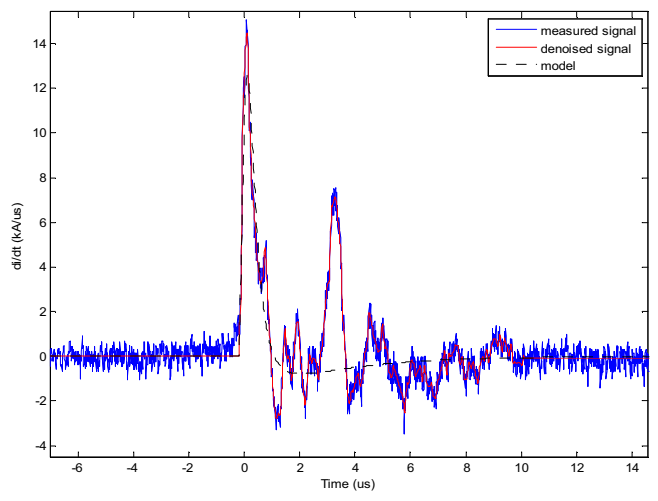

Figure 4. Denoised current derivative waveform and its Heidler model

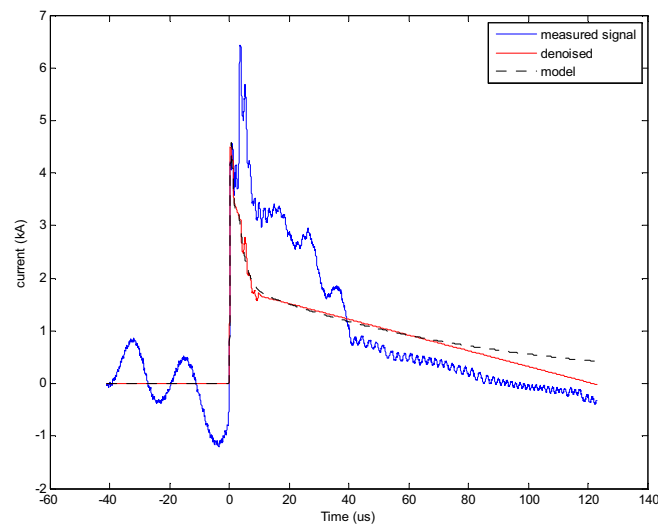

Figure 5. Denoised current waveform and its Heidler generated model ( mismatch of the current derivative with its model)

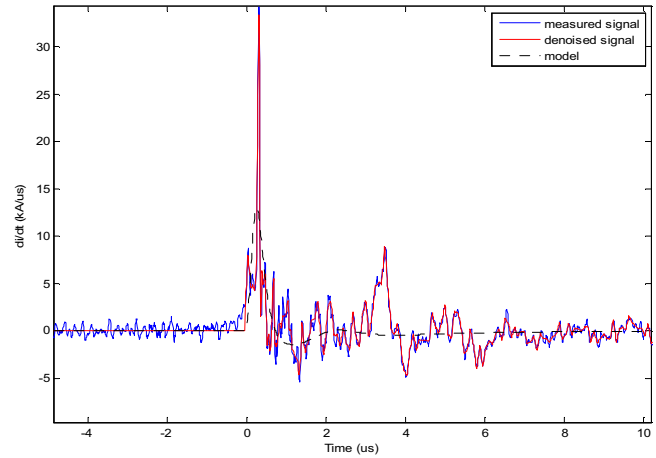

Figure 6. Denoised current derivative waveform and its Heidler generated model (mismatch of the current derivative with its model)

TABle 1. CuRrent DeRIVATIVE PARAMETERS

\begin{tabular}{|c|c|c|c|}
\hline $\begin{array}{c}\text { Max } \\
\text { Peak } \\
(\mathbf{k A} / \boldsymbol{\mu} \mathbf{s})\end{array}$ & $\begin{array}{c}\text { Max } \\
\text { steepness } \\
\left(\mathbf{k A} / \boldsymbol{\mu} \mathbf{s}^{2}\right)\end{array}$ & $\begin{array}{c}\text { Rise } \\
\text { time } \\
(\boldsymbol{\mu s})\end{array}$ & $\begin{array}{c}\mathbf{5 0 \%} \\
\text { width } \\
(\boldsymbol{\mu s})\end{array}$ \\
\hline 14.9 & 321.0 & 0.1 & 0.3 \\
\hline
\end{tabular}

TABle 2. CuRRENT PARAMETERS

\begin{tabular}{|c|c|c|c|c|}
\hline $\begin{array}{c}\text { Max } \\
\text { Peak } \\
(\mathbf{k A})\end{array}$ & $\begin{array}{c}\text { Max } \\
\text { steepness } \\
(\mathbf{k A} / \boldsymbol{\mu s})\end{array}$ & $\begin{array}{c}\text { Rise } \\
\text { time } \\
(\boldsymbol{\mu s})\end{array}$ & $\begin{array}{c}\mathbf{5 0 \%} \\
\text { widt } \\
\mathbf{h} \\
(\boldsymbol{\mu s})\end{array}$ & $\begin{array}{c}\text { total } \\
\text { charge } \\
(\mathbf{C})\end{array}$ \\
\hline 7.7 & 14.9 & 0.7 & 53.6 & 208.6 \\
\hline
\end{tabular}

TABLE 3. HEIDLER MODEL PARAMETERS

\begin{tabular}{|l|l|l|l|l|l|l|}
\hline $\begin{array}{l}\boldsymbol{I}_{\boldsymbol{l}} \\
(\boldsymbol{k A})\end{array}$ & $\begin{array}{l}\boldsymbol{I}_{2} \\
(\boldsymbol{k A})\end{array}$ & $\boldsymbol{K}_{1,2}$ & $\begin{array}{l}\tau_{1,1} \\
(\mu s\end{array}$ & $\begin{array}{l}\tau_{1,2} \\
(\mu s \\
)\end{array}$ & $\begin{array}{l}\tau_{2,1} \\
(\mu s\end{array}$ & $\begin{array}{l}\tau_{2,2} \\
(\mu s \\
)\end{array}$ \\
\hline 15 & 7.7 & 2.2 & 0.5 & 5.0 & 9.9 & 56 \\
\hline
\end{tabular}

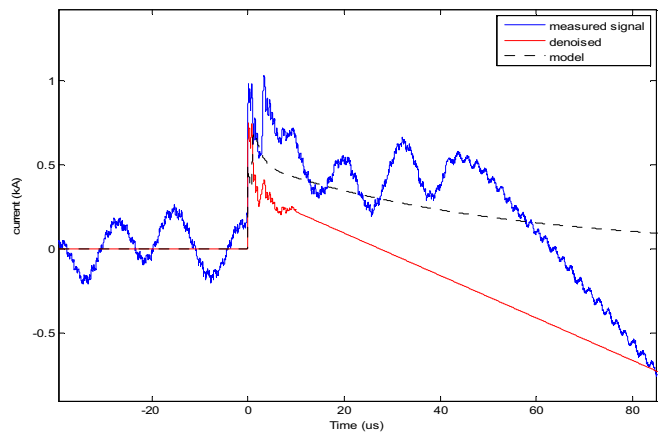

Figure 7. Denoised current waveform and its Heidler generated model ( mismatch of the current derivative with its model) 


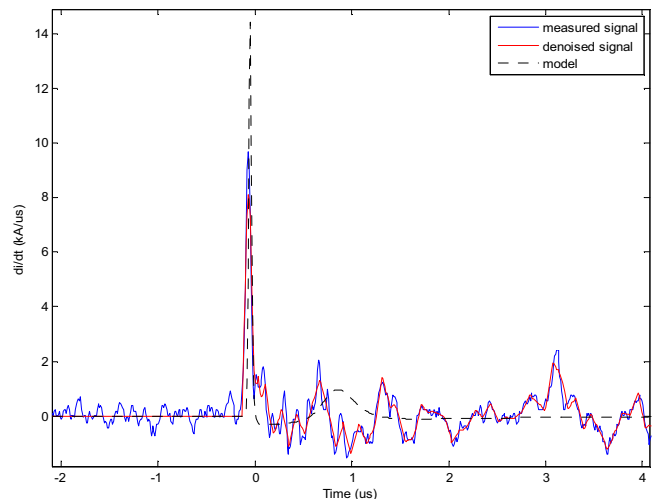

Figure 8. Denoised current derivative waveform and its Heidler generated model (mismatch of the current derivative with its model)

\section{Conclusion}

Not only has the newly developed DAC denoising approach allowed the calculation of the parameters of the lightning current derivative waveforms measured at the $\mathrm{CN}$ tower and their associated current waveforms with high precision, it also allowed the generation of models for the measured waveforms by the use of Heidler function. These models can be represented only by their 10 parameters. This brings the transmission of the lengthy (from 30 Kbytes to 1 Mbytes) and noisy lightning current waveforms to the LDN stations to the transmission of just their $2 \times 10$ bytes (two bytes each parameter) Heidler model parameters. Finding that not all the optimized models are representative of their corresponding measured waveforms (interaction of more than one lightning strike), other criteria have been selected for the models to be transmitted to the LDN stations. These constraints consist in imposing for the models waveforms parameters to match the ones of the measured waveforms, as the wavefront peaks, the rise time to the peaks and the widths at $50 \%$ of the peaks. More constraints can be added as the satisfaction of a high correlation factor between the denoised waveforms and the models.

\section{References}

[1] Martin A. Uman, “The Lightning Discharge," Dover Publication, Inc., Mineola, New York, 2001.

[2] A.M. Hussein, W. Janischewskyj, J.-S. Chang, V. Shostack, W.A. Chisholm, P. Dzurevych, and Z.-I. Kawasaki, "Simultaneous measurement of lightning parameters for strokes to the Toronto Canadian National
Tower," Journal of Geophysical Research-Atmosphere, vol.100, no. 5, pp. 8853-8861, May 1995.

[3] W. Janischewskyj, A.M Hussein, "Statistics of Lightning Strikes to the Toronto Canadian National Tower (1978-1995)," IEEE Trans., Power Delivery, vol. 12, pp. 1210-1221, No. 3, July 1997.

[4] A.M. Hussein, W. Janischewskyj, M. Milewski, V. Shostak, J.S. Chang and W. Chisholm, "Current waveform parameters of $\mathrm{CN}$ Tower lightning return strokes," Journal of Electrostatics, Vol. 60, Nos. 2-4, pp. 149-162, March 2004.

[5] A.M. Hussein, K. Bitner and M. Milewski, "Modelling of CN Tower's Lightning Return Stroke Current Using the Derivative of the Heidler Function," Proceedings of the 28th International Conference on Lightning Protection (ICLP), Kanazawa, Japan, pp. 261-266, September, 18-22, 2006.

[6] Nicos herodotou, "Study of Currents Due to lightning in Ontario Using an LLP System", Master thesis, University of Toronto, 1990.

[7] P. Liatos and A.M. Hussein, "Characterization of Noise in the Lightning Current Derivative Signals Measured at the CN Tower," IEEE Transaction on Electromagnetic Compatibility (EMC), vol. 47, No. 4, pp. 986-997, November, 2005.

[8] O. Nedjah, A.M. Hussein, R. Sotudeh and W. Janischewskyj, "Wavelet Noise Removal From CN Tower Lightning Current Waveforms," International Signal Processing Conference Paper 505 (pp. 1-6), Dallas, Texas, Mar. 31-Apr.4, 2003.

[9] O. Nedjah and Ali M. Hussein, "CN Tower lightning current de-noising: wavelet transform versus an optical fiber link," International Conference for Upcoming Engineers (ICUE), Ryerson University, Toronto, Ontario, Canada, May 13-14, 2004, Paper \#06.

[10] O. Nedjah and Ali M. Hussein, R. Sotudeh, Sri Krishnan "CN Tower Lightning Current Derivative Heidler Model for the Validation of Wavelet De-Noising Algorithm,"Proceedings, 18th International Wroclaw Symposium and Exhibition on Electromagnetic Compatibility, pp. 282 - 287, Wroclaw, Poland, June 28 $-30,2006$.

[11] O.Nedjah , Ali Hussein, Sri Krishnan, R. Sotudeh, Kaamran Raahemifar, "A Divide-and-Conquer Approach for Denoising and Modeling The CN Tower Lightning Current Derivative Signal", IEEE Canadian Conference on Electical and Computer Engineering, Paper 93493 (PP. 1373-1378), Niagara Falls, May 4-7, 2008.

[12] Ying Chen, "Wavelet Analysis and Statistics of CN Tower Lightning Current Waveforms," M.E.Sc. Thesis, University of Weston Ontario, London, Ontario, 1997.

[13] F. Heidler, J.M. Cvetic, B.V. Stanic, "Calculation of lightning Current Parameters", IEEE transaction on Power delivery, Vol. 14, No. 2, pp. 399-404, April 1999.

[14] Greg Astfalk, Irvin Lustig, Roy Marsten, David Shanno, "The interior-point method for Linear Programming," IEEE software, pp. 61-68, July 1992. 